\title{
Two general theorems on superstability of functional equations
}

\author{
Janusz BrzDesk, AdAm NAJdecki And Bing XU
}

\begin{abstract}
We prove that the superstability of some functional equations (e.g., of Cauchy, d'Alembert, Wilson, Reynolds, and homogeneity) is a consequences of two simple theorems. In this way we generalize several classical superstability results.
\end{abstract}

Mathematics Subject Classification (2010). 39B82.

Keywords. Superstability, functional equation.

Throughout this note, $\mathbb{N}, \mathbb{R}$ and $\mathbb{C}$ stand, as usual, for the sets of positive integers, reals and complex numbers, respectively. Moreover $\mathbb{N}_{0}:=\mathbb{N} \cup\{0\}, \mathbb{K}$ is either the field $\mathbb{R}$ or $\mathbb{C}, X$ is a normed linear space over $\mathbb{K}$, and $S$ and $T$ are nonempty sets.

We show that, under suitable assumptions, unbounded solutions of some functional inequalities actually must be solutions of the corresponding functional equations. This phenomenon is connected with the well known problem of stability of functional equations, which started with a question of Ulam and Hyers solution to it (see [17]; for more details see [15,18,21]). It seems that for the first time it has been discovered by Baker et al. [6], during the study of the stability of the exponential equation

$$
f(x+y)=f(x) f(y) .
$$

Namely, they have proved that if $f$ is a function mapping a real vector space $X$ into $\mathbb{R}$, satisfying

$$
|f(x+y)-f(x) f(y)| \leq \delta, \quad \forall x, y \in X
$$

with some real $\delta>0$, then $f$ is either bounded or exponential. That property of functional equations is now usually called superstability and has already been investigated in numerous papers (see, e.g., [2-5, 7,9-11,14, 16, 19, 20,28-35]) for numerous functional equations. 
Let $a_{j} \in \mathbb{K}$ and $g_{j}: T \times S \rightarrow S$ for $j \in \mathbb{N}$. Looking closer at the known results concerning the superstability of various particular cases of the equation

$$
\sum_{j=1}^{\infty} a_{j} f\left(g_{j}(t, s)\right)=h(t) f(s)
$$

(with unknown functions $f: S \rightarrow X$ and $h: T \rightarrow \mathbb{K}$ ) one may notice that there are many similarities between the ideas they are based on. Therefore we could expect some more general theorems from which many of those superstability outcomes could be derived. In this paper we prove two such simple theorems and show examples of their consequences.

We write

$$
\mathcal{F}:=\left\{f: S \rightarrow X: \sum_{j=1}^{\infty} a_{j} f\left(g_{j}(t, s)\right) \text { is uniformly convergent in } T \times S\right\} .
$$

Clearly $\mathcal{F}=X^{S}$, if there is $M \in \mathbb{N}$ such that $a_{j}=0$ for $j>M$.

Theorem 1. Suppose that $h: T \rightarrow \mathbb{K}, f \in \mathcal{F}, N \in \mathbb{N}, \varepsilon_{j}: S \rightarrow \mathbb{R}$ for $j \in \mathbb{N}, \delta>0$,

$$
\begin{array}{r}
\left\|\sum_{j, k=1}^{l} a_{j} a_{k}\left[f\left(g_{j}\left(t_{2}, g_{k}\left(t_{1}, s\right)\right)\right)-f\left(g_{k}\left(t_{1}, g_{j}\left(t_{2}, s\right)\right)\right)\right]\right\| \leq \varepsilon_{l}(s), \\
\forall t_{1}, t_{2} \in T, s \in S, l \in \mathbb{N}, l \geq N
\end{array}
$$

and

$$
\left\|\sum_{j=1}^{\infty} a_{j} f\left(g_{j}(t, s)\right)-h(t) f(s)\right\| \leq \delta, \quad \forall t \in T, s \in S .
$$

Then one of the following two conditions holds.

(i) $h$ is bounded.

(ii) $h$ and $f$ satisfy Eq. (2) and there is $M \in \mathbb{N}$ such that

$$
a_{j} f\left(g_{j}(t, s)\right)=0, \quad \forall s \in S, t \in T, j>M .
$$

Proof. Assume that $h$ is unbounded. Since $f \in \mathcal{F}$, there exists $M \in \mathbb{N}, M \geq N$, such that

$$
\left\|\sum_{j=M+m}^{\infty} a_{j} f\left(g_{j}(t, s)\right)\right\| \leq \delta, \quad \forall t \in T, s \in S, m \in \mathbb{N}_{0} .
$$

Consequently, for each $t \in T, s \in S, m \in \mathbb{N}_{0}$,

$$
\begin{aligned}
\left\|\sum_{j=1}^{M+m} a_{j} f\left(g_{j}(t, s)\right)-h(t) f(s)\right\| \leq & \left\|\sum_{j=1}^{\infty} a_{j} f\left(g_{j}(t, s)\right)-h(t) f(s)\right\| \\
& +\left\|\sum_{j=M+m+1}^{\infty} a_{j} f\left(g_{j}(t, s)\right)\right\| \leq 2 \delta .
\end{aligned}
$$


Let $\left(t_{n}: n \in \mathbb{N}\right)$ be a sequence in $T$ with $h\left(t_{n}\right) \neq 0$ for $n \in \mathbb{N}$ and $\lim _{n \rightarrow \infty}\left|h\left(t_{n}\right)\right|=\infty$. Then (6) implies that

$$
f(s)=\lim _{n \rightarrow \infty} \sum_{i=1}^{M+m} \frac{a_{i}}{h\left(t_{n}\right)} f\left(g_{i}\left(t_{n}, s\right)\right), \quad \forall s \in S, m \in \mathbb{N}_{0} .
$$

Next, replacing $s$ by $g_{k}\left(t_{n}, s\right)$ in (6), we obtain

$$
\begin{aligned}
& \left\|\sum_{j=1}^{M+m} a_{j} f\left(g_{j}\left(t, g_{k}\left(t_{n}, s\right)\right)\right)-h(t) f\left(g_{k}\left(t_{n}, s\right)\right)\right\| \\
& \quad \leq 2 \delta, \quad \forall t \in T, s \in S, m \in \mathbb{N}_{0}, k \in \mathbb{N} .
\end{aligned}
$$

Consequently, using (7), (3) and (8), for $t \in T, s \in S, m \in \mathbb{N}_{0}$, we get

$$
\begin{aligned}
& \sum_{j=1}^{M+m} a_{j} f\left(g_{j}(t, s)\right) \\
& =\sum_{j=1}^{M+m} a_{j} \lim _{n \rightarrow \infty} \frac{1}{h\left(t_{n}\right)} \sum_{k=1}^{M+m} a_{k} f\left(g_{k}\left(t_{n}, g_{j}(t, s)\right)\right) \\
& =\lim _{n \rightarrow \infty} \frac{1}{h\left(t_{n}\right)} \sum_{k=1}^{M+m} a_{k} \sum_{j=1}^{M+m} a_{j} f\left(g_{j}\left(t, g_{k}\left(t_{n}, s\right)\right)\right) \\
& =\lim _{n \rightarrow \infty} \frac{1}{h\left(t_{n}\right)} \sum_{k=1}^{M+m} a_{k}\left[\sum_{j=1}^{M+m} a_{j} f\left(g_{j}\left(t, g_{k}\left(t_{n}, s\right)\right)\right)-h(t) f\left(g_{k}\left(t_{n}, s\right)\right)\right] \\
& \quad+\lim _{n \rightarrow \infty} h(t) \sum_{k=1}^{M+m} \frac{a_{k}}{h\left(t_{n}\right)} f\left(g_{k}\left(t_{n}, s\right)\right)=h(t) f(s),
\end{aligned}
$$

which implies (5). Hence $f$ and $h$ satisfy Eq. (2).

Corollary 1. Assume that $N \in \mathbb{N}, \delta>0, X=\mathbb{K}, S=T$, (3) holds, and $f \in \mathcal{F}$ satisfies

$$
\left|\sum_{j=1}^{\infty} a_{j} f\left(g_{j}(t, s)\right)-f(t) f(s)\right| \leq \delta, \quad \forall s, t \in S .
$$

Then one of the following two conditions holds.

(i) There is $M \in \mathbb{N}$ such that (5) holds and $f$ is an unbounded solution of the equation

$$
\sum_{j=1}^{\infty} a_{j} f\left(g_{j}(t, s)\right)=f(t) f(s) .
$$


(ii) $f$ is bounded and, in the case $a:=\sum_{j=1}^{\infty}\left|a_{j}\right|<\infty$,

$$
|f(x)| \leq \frac{a+\sqrt{a^{2}+4 \delta}}{2}, \quad \forall x \in S .
$$

Proof. In view of Theorem 1 it is enough to consider the case when $f$ is bounded. Let

$$
\sigma:=\sup \{|f(x)|: x \in S\} .
$$

Then there exists a sequence $\left(y_{n}: n \in \mathbb{N}\right)$ of elements of $S$ such that $\lim _{n \rightarrow \infty}$ $\left|f\left(y_{n}\right)\right|=\sigma$, and for $n \in \mathbb{N}$

$$
\left|f\left(y_{n}\right)\right|^{2} \leq \delta+\sum_{j=1}^{\infty}\left|a_{j}\right|\left|f\left(g_{j}\left(y_{n}, y_{n}\right)\right)\right| \leq \delta+a \sigma .
$$

Letting $n \rightarrow \infty$ we have $\sigma^{2}-a \sigma-\delta \leq 0$, so

$$
\sigma \leq \frac{a+\sqrt{a^{2}+4 \delta}}{2}
$$

Remark 1. Actually, as it is easily seen in the proof of Theorem 1 (and Corollary 1), the assumption that $f \in \mathcal{F}$ can be weakened. Namely it is enough to assume that there is $M \in \mathbb{N}$ such that, for each $k \in \mathbb{N}, k \geq M$, there is $L_{k} \in \mathbb{R}$ with

$$
\left\|\sum_{j=k}^{\infty} a_{j} f\left(g_{j}(t, s)\right)\right\| \leq L_{k}, \quad \forall t \in T, s \in S .
$$

The same applies to the next Theorem 2 and Corollary 2.

Remark 2. If $\sum_{i=1}^{\infty} a_{i}=\sum_{i=1}^{\infty}\left|a_{i}\right|$, then estimation (11) is sharp, because (3) and (9) hold with $f(t)=\frac{1}{2}\left(a+\sqrt{a^{2}+4 \delta}\right)$ for $t \in S$.

Remark 3. One can easily see that estimation (11) in particular cases is the same as obtained by Baker in [5] for the exponential functional equation (if $a_{1}=1$ and $a_{j}=0$ for $j>1$ ) and d'Alembert's equation (if $a_{1}=a_{2}=\frac{1}{2}$ and $a_{j}=0$ for $j>2$ ).

Example 1. Below we give some examples of functions $g_{i}$ satisfying (3).

(i) Let $(G,+)$ be an abelian semigroup, $\lambda: S \rightarrow G$ be surjective, and $\varrho$ : $G \rightarrow S$ be a selection of $\lambda$ (i.e., $\lambda(\varrho(x))=x$ for $x \in G)$. Let $\gamma_{j}: T \rightarrow G$ and $g_{j}(t, s)=\varrho\left(\gamma_{j}(t)+\lambda(s)\right)$ for $j \in \mathbb{N}, t \in T, s \in S$. Then (3) holds for any $f \in X^{S}$ (with $N=1$ and $\varepsilon_{l}(s)=0$ for $s \in S$ ). 
In the simplified case: $S=G$ and $\lambda(s)=s$ for $s \in S$, Eqs. (2) and (10) take the forms

$$
\begin{aligned}
& \sum_{j=1}^{\infty} a_{j} f\left(\gamma_{j}(t)+s\right)=h(t) f(s), \\
& \sum_{j=1}^{\infty} a_{j} f\left(\gamma_{j}(t)+s\right)=f(t) f(s) .
\end{aligned}
$$

The following functional equations are particular cases of them: the exponential Cauchy Eq. (1) and the multiplicative Cauchy equation $f(x y)=$ $f(x) f(y)$, their partially pexiderized versions $f(x y)=f(x) h(y)$ and $f(x+$ $y)=f(x) h(y)$, d'Alembert's equation

$$
f(x+y)+f(x-y)=2 f(x) f(y)
$$

and its generalized version $f(x+y)+f(x+\tau(y))=f(x) h(y)$ (considered in $[4,22]$; see also Example 2), the functional equation

$$
f(x+f(y))=f(x) f(y)
$$

(which is related with the Reynolds operator; see, e.g., [13,25]) and the following pexiderization of it: $f(x+g(y))=h(y) f(x)$. The superstability of Eq. (14) has been proved in [27].

(ii) Let $Y$ be a module over a commutative $\operatorname{ring} \mathbb{F}, \xi: S \rightarrow Y$ be a surjection, $\varrho: Y \rightarrow S$ be a selection of $\xi$, and $\gamma_{j}: T \rightarrow \mathbb{F}$ for $j \in \mathbb{N}$. Let $g_{j}(t, s)=$ $\varrho\left(\gamma_{j}(t) \xi(s)\right)$ for $t \in T, s \in S$. Then it is easy to check that (3) holds for any $f \in X^{S}$ (with $N=1$ and $\varepsilon_{l}(s)=0$ for $s \in S$ ).

In the simplified case: $S=Y$ and $\xi(s)=s$ for $s \in S$, Eq. (2) takes the form

$$
\sum_{j=1}^{\infty} a_{j} f\left(\gamma_{j}(t) s\right)=h(t) f(s)
$$

the superstability of the following particular case of it, the homogeneity functional equation $f(\gamma(t) s)=h(t) f(s)$, has been investigated, e.g., in $[19,20,34,35]$. Clearly, the homogeneity equation may be considered a pexiderization of the equation $f(f(x) y)=f(x) f(y)$, also connected with the Reynolds operator; in [8] it has been suggested to name that last equation: the Reynolds equation.

(iii) Let $\xi: S \rightarrow(0, \infty)$ be a surjection, $\varrho:(0, \infty) \rightarrow S$ be a selection of $\xi$, and $\gamma_{j}: T \rightarrow \mathbb{R}$ for $j \in \mathbb{N}$. Let $g_{j}(t, s)=\varrho\left(\xi(s)^{\gamma_{j}(t)}\right)$ for $s \in S, t \in T$. Then it is easy to check that (3) holds for any $f \in X^{S}$ (with $N=1$ and $\varepsilon_{l}(s)=0$ for $\left.s \in S\right)$. 
If $S=(0, \infty)$ and $\xi(s)=s$ for $s \in S$, Eq. (2) takes the form

$$
\sum_{j=1}^{\infty} a_{j} f\left(s^{\gamma_{j}(t)}\right)=h(t) f(s)
$$

and the special case of it is the functional equation $f\left(s^{t}\right)=t f(s)$; its superstability has been proved in $[20,35]$.

(iv) Let $(V, \cdot)$ be a groupoid (i.e., $V$ is a nonempty set endowed with a binary operation $\cdot: G^{2} \rightarrow G$ ) with $\left(a^{n}\right)^{m}=a^{m n}$ for $a \in V, m, n \in \mathbb{N}$ (where $a^{1}=a$ and $a^{n+1}=a^{n} \cdot a$ for $\left.n \in \mathbb{N}\right), \gamma_{j}: T \rightarrow \mathbb{N}$ for $j \in \mathbb{N}, \xi: S \rightarrow V$ be a surjection and $\varrho: V \rightarrow S$ be a selection of $\xi$. Write $g_{j}(t, s)=\varrho\left(\xi(s)^{\gamma_{j}(t)}\right)$ for $j \in \mathbb{N}, t \in T, s \in S$. Then it is easy to check that (3) holds for any $f \in X^{S}$ (with $N=1$ and $\varepsilon_{l}(s)=0$ for $\left.s \in S\right)$. Clearly, if $(V, \cdot)$ is a group, then we can take $\gamma_{j}: T \rightarrow \mathbb{Z}$.

(v) Let $(G,+)$ be an abelian groupoid and $g: G \times S \rightarrow S$ be a solution of the translation equation $g\left(t_{1}, g\left(t_{2}, s\right)\right)=g\left(t_{1}+t_{2}, s\right)$ (for more information on the translation equation see, e.g., [26]). Clearly $g\left(t_{2}, g\left(t_{1}, s\right)\right)=$ $g\left(t_{1}, g\left(t_{2}, s\right)\right)$ for every $t_{1}, t_{2} \in G, s \in S$, which means that (3) holds for any $f \in X^{S}$ (with $N=1$ and $\varepsilon_{l}(s)=0$ for $s \in S$ ).

(vi) The last example is somewhat trivial. Namely, let $\lambda_{i}: S \rightarrow S$ and $g_{i}(t, s)=\lambda_{i}(s)$ for $i \in \mathbb{N}, t, s \in S$. Then (3) holds with $N=1$ and $\varepsilon_{l}(s)=\left\|\sum_{i, j=1}^{l} a_{i} a_{j}\left[f\left(\lambda_{j}\left(\lambda_{i}(s)\right)\right)-f\left(\lambda_{i}\left(\lambda_{j}(s)\right)\right)\right]\right\|$ for $l \in \mathbb{N}, s \in S$.

Note that in this case, for any $r \in S$ with $f(r) \neq 0$, inequality (4) implies that

$$
|h(t)| \leq \frac{1}{\|f(r)\|}\left(\delta+\left\|\sum_{j=1}^{\infty} a_{j} f\left(\lambda_{j}(r)\right)\right\|\right), \quad \forall t \in T,
$$

whence $f(s)=0$ for each $s \in S$ or $h$ is bounded.

The next theorem concerns the case $T=S$.

Theorem 2. Let $X$ be locally compact, $T=S, N \in \mathbb{N}, g_{i}: S^{2} \rightarrow S$ and $\varepsilon_{i}: S^{2} \rightarrow \mathbb{R}$ for $i \in \mathbb{N}$, and $\delta: S \rightarrow \mathbb{R}$. Suppose that $f \in \mathcal{F}$ and $h: S \rightarrow \mathbb{K}$ satisfy the inequalities

$$
\begin{aligned}
& \left\|\sum_{i, j=1}^{l} a_{i} a_{j}\left[f\left(g_{i}\left(t, g_{j}(s, r)\right)\right)-f\left(g_{i}\left(g_{j}(t, s), r\right)\right)\right]\right\| \\
& \quad \leq \varepsilon_{l}(t, s), \quad \forall t, s, r \in S, l \in \mathbb{N}, l \geq N, \\
& \left\|\sum_{i=1}^{\infty} a_{i} f\left(g_{i}(t, s)\right)-h(t) f(s)\right\| \leq \delta(t), \quad \forall t, s \in S .
\end{aligned}
$$

Then the following two conditions hold. 
(i) If $f$ is unbounded, then $h$ is a solution of the functional equation

$$
\sum_{j=1}^{\infty} a_{j} h\left(g_{j}(t, s)\right)=h(t) h(s), \quad \forall t, s \in S
$$

and there exists $M \in \mathbb{N}$ with $a_{j} h\left(g_{j}(t, s)\right)=0$ for $t, s \in S, j>M$.

(ii) If $0<C:=\sup _{s \in S}\|f(s)\|<\infty$ and $a:=\sum_{j=1}^{\infty}\left|a_{j}\right|<\infty$, then

$$
|h(t)| \leq a+\frac{\delta(t)}{C}, \quad \forall t \in S .
$$

Proof. First assume that $C:=\sup _{s \in S}\|f(s)\|<\infty$ and $a:=\sum_{j=1}^{\infty}\left|a_{j}\right|<\infty$. Then, for every $s, t \in S$,

$$
\|h(t) f(s)\| \leq\left\|\sum_{i=1}^{\infty} a_{i} f\left(g_{i}(t, s)\right)\right\|+\delta(t) \leq a C+\delta(t),
$$

whence $|h(t)| C \leq a C+\delta(t)$ and consequently we obtain (18).

Now, suppose that $f$ is unbounded and take a real $L>0$. Since $f \in \mathcal{F}$, there exists $M(L) \in \mathbb{N}, M(L) \geq N$, such that

$$
\left\|\sum_{i=M(L)+m}^{\infty} a_{i} f\left(g_{i}(t, s)\right)\right\| \leq L, \quad \forall t, s \in S, m \in \mathbb{N}_{0} .
$$

Hence

$$
\begin{aligned}
& \left\|\sum_{i=1}^{M(L)+m} a_{i} f\left(g_{i}(t, s)\right)-h(t) f(s)\right\| \leq\left\|\sum_{i=1}^{\infty} a_{i} f\left(g_{i}(t, s)\right)-h(t) f(s)\right\| \\
& +\left\|\sum_{i=M(L)+m+1}^{\infty} a_{i} f\left(g_{i}(t, s)\right)\right\| \leq \delta(t)+L, \quad \forall s, t \in S, m \in \mathbb{N}_{0} .
\end{aligned}
$$

Let $\left(s_{n}: n \in \mathbb{N}\right)$ be a sequence in $S$ with $f\left(s_{n}\right) \neq 0$ for $n \in \mathbb{N}$ and $\lim _{n \rightarrow \infty}\left\|f\left(s_{n}\right)\right\|=\infty$. Since $X$ is locally compact and consequently the unit ball in $X$ is compact, without loss of generality (it is enough to replace $\left(s_{n}\right.$ : $n \in \mathbb{N}$ ) by a suitable subsequence) we can assume that there is $x \in X$ with $\|x\|=1$ and $x=\lim _{n \rightarrow \infty} \frac{1}{\left\|f\left(s_{n}\right)\right\|} f\left(s_{n}\right)$. So, by $(19)$,

$$
h(t) x=\lim _{n \rightarrow \infty} \frac{1}{\left\|f\left(s_{n}\right)\right\|} \sum_{i=1}^{M(L)+m} a_{i} f\left(g_{i}\left(t, s_{n}\right)\right), \quad \forall m \in \mathbb{N}_{0}, t \in S .
$$

Further, (15) and (19) imply that, for every $u, v \in S, m \in \mathbb{N}_{0}$ with $m \geq$ $M(L)$, 


$$
\begin{aligned}
& \left\|\sum_{i, j=1}^{m} a_{i} a_{j} f\left(g_{i}\left(g_{j}(u, v), s_{n}\right)\right)-h(u) \sum_{j=1}^{m} a_{j} f\left(g_{j}\left(v, s_{n}\right)\right)\right\| \leq \varepsilon_{m}(u, v) \\
& \quad+\left\|\sum_{i, j=1}^{m} a_{i} a_{j} f\left(g_{i}\left(u, g_{j}\left(v, s_{n}\right)\right)\right)-h(u) \sum_{j=1}^{m} a_{j} f\left(g_{j}\left(v, s_{n}\right)\right)\right\| \\
& \quad \leq \varepsilon_{m}(u, v)+\sum_{j=1}^{m}\left|a_{j}\right|\left\|\sum_{i=1}^{m} a_{i} f\left(g_{i}\left(u, g_{j}\left(v, s_{n}\right)\right)\right)-h(u) f\left(g_{j}\left(v, s_{n}\right)\right)\right\| \\
& \leq \varepsilon_{m}(u, v)+(\delta(u)+L) \sum_{j=1}^{m}\left|a_{j}\right|
\end{aligned}
$$

and consequently

$$
\begin{aligned}
& \left\|\sum_{j=1}^{m} a_{j} \frac{\sum_{i=1}^{m} a_{i} f\left(g_{i}\left(g_{j}(u, v), s_{n}\right)\right)}{\left\|f\left(s_{n}\right)\right\|}-h(u) \frac{\sum_{i=1}^{m} a_{i} f\left(g_{i}\left(v, s_{n}\right)\right)}{\left\|f\left(s_{n}\right)\right\|}\right\| \\
& \quad \leq \frac{1}{\left\|f\left(s_{n}\right)\right\|}\left(\varepsilon_{m}(u, v)+(\delta(u)+L) \sum_{i=1}^{m}\left|a_{j}\right|\right) .
\end{aligned}
$$

Letting $n \rightarrow \infty$ we obtain

$$
\sum_{j=1}^{m} a_{j} h\left(g_{j}(u, v)\right)=h(u) h(v) \quad \forall u, v \in S, m \in \mathbb{N}_{0}, m \geq M(L) .
$$

Clearly, this yields $a_{j} h\left(g_{j}(t, s)\right)=0$ for $t, s \in S, j>M(L)$.

Thus we have proved that $h$ is a solution of Eq. (17).

Remark 4. In the situation where $\sum_{i=1}^{\infty} a_{i}=\sum_{i=1}^{\infty}\left|a_{i}\right|$, estimation (18) is sharp, because (15) and (16) hold for $h(t)=a+\frac{\delta(t)}{C}$ and $f(t)=x_{0}$ for $t \in S$, with every $x_{0} \in X,\left\|x_{0}\right\|=C$.

We also have the subsequent.

Corollary 2. Assume that $T=S, N \in \mathbb{N}, g_{i}: S^{2} \rightarrow S$ and $\varepsilon_{i}: S^{2} \rightarrow \mathbb{R}$ for $i \in \mathbb{N}, \delta: S \rightarrow \mathbb{R}, X=\mathbb{K},(15)$ holds, and $f \in \mathcal{F}$ satisfies

$$
\left|\sum_{j=1}^{\infty} a_{j} f\left(g_{j}(t, s)\right)-f(t) f(s)\right| \leq \delta(t), \quad \forall s, t \in S .
$$

Then the following two conditions hold.

(i) If $f$ is unbounded, then it is a solution of Eq. (17) and there exists $M \in \mathbb{N}$ with $a_{j} f\left(g_{j}(t, s)\right)=0$ for $t, s \in S, j>M$.

(ii) If $0<C:=\sup _{s \in S}|f(s)|<\infty$ and $a:=\sum_{j=1}^{\infty}\left|a_{j}\right|<\infty$, then

$$
|f(t)| \leq a+\frac{\delta(t)}{C}, \quad \forall t \in S
$$


moreover, in the case $\Delta:=\sup _{s \in S} \delta(s)<\infty$,

$$
C \leq \frac{a+\sqrt{a^{2}+4 \Delta}}{2} .
$$

Proof. It is enough to use Theorem 2 with $h=f$ and argue as in the proof of Corollary 1.

Remark 5. If $\sum_{i=1}^{\infty} a_{i}=\sum_{i=1}^{\infty}\left|a_{i}\right|$, then without additional assumptions estimation (23) cannot be improved, because (15) and (21) hold with $f(t)=$ $\frac{1}{2}\left(a+\sqrt{a^{2}+4 \Delta}\right)$ and $\delta(t)=\Delta$ for $t \in S$.

It seems that it is also the case for (22), for take $a, C \in(0, \infty), a<C, S:=$ $[a, C], g_{1}(t, s):=s, f(t):=t$ and $\delta(t):=C(t-a)$ for $t, s \in S$. Then it is easy to check that (15) and (21) are valid with $a_{1}:=a$ and $a_{j}:=0$ (and any $g_{j}$ ) for $j>1$.

Example 2. (i) Let $(S,+)$ be an abelian semigroup, $c_{i} \in S$ and $g_{i}(t, s)=$ $t+s+c_{i}$ for $i \in \mathbb{N}, t, s \in S$. Then (15) holds for any $f \in X^{S}$ (with $N=1$ and $\varepsilon_{l}(t, s)=0$ for $t, s \in S$ ) and functional Eq. (2) has the form

$$
\sum_{i=1}^{\infty} a_{i} f\left(t+s+c_{i}\right)=h(t) f(s) ;
$$

in particular, (17) has then the form (see [33]): $\sum_{i=1}^{\infty} a_{i} h\left(t+s+c_{i}\right)=$ $h(t) h(s)$.

(ii) Let $a_{1}=a_{2}, a_{j}=0$ for $j>2,(S,+)$ be an abelian semigroup, $\tau: S \rightarrow S$ be a homomorphism with $\tau(\tau(s))=s$ for $s \in S$. Then, in either of the following two cases:

- $g_{1}(t, s)=t+s, g_{2}(t, s)=\tau(t)+s$ for $t, s \in S$;

- $g_{1}(t, s)=t+s, g_{2}(t, s)=t+\tau(s)$ for $t, s \in S$,

condition (15) holds for any $f \in X^{S}$ (with $N=1$ and $\varepsilon_{l}(t, s)=0$ for $t, s \in S$ ), and Eq. (2) takes (with $a_{1}=1$ ) one of the forms:

$$
\begin{gathered}
f(t+s)+f(\tau(t)+s)=h(t) f(s), \\
f(t+s)+f(t+\tau(s))=h(t) f(s) .
\end{gathered}
$$

Those two equations are partial pexiderizations of a generalization of the d'Alembert functional Eq. (13). Their superstability has been discussed in $[4,22]$; one can easily derive [4, Theorems 1,2$]$ and $[22$, Theorems 1,4$]$ from our Theorem 2; independently, results analogous to those in [22], with $\tau(s)=-s$, have been obtained by Tyrala [36]. [In the case $\tau(s)=-s$, Eq. (25) is known as Wilson's first generalization of d'Alembert's functional equation and Eq. (26) is a particular case of Wilson's second generalization of d'Alembert's functional equation (see [1]; cf. also [37])].

(iii) In the case where $a_{1}=1$ and $a_{j}=0$ for $j>1,(S, \cdot)$ is a semigroup, $\mu: S \rightarrow S$ is a homomorphism with $\mu \circ \mu=\mu$, and $g_{1}(t, s)=\mu(t) \cdot \mu(s)$, 
condition (15) holds for every $f: S \rightarrow X$ (with $N=1$ and $\varepsilon_{l}(t, s)=0$ for $t, s \in S$ ) and, if in particular $\mu(s)=s$ for $s \in S$, (2) has the form $f(t \cdot s)=h(t) f(s)$, which is a partial pexiderization of the equation $h(t \cdot s)=$ $h(t) h(s)$. In this case, from Theorem 2 , we obtain a new characterization of multiplicative functions $h: S \rightarrow \mathbb{K}$.

(iv) Let $(G, \star)$ be a semigroup, $\lambda: S \rightarrow G$ be surjective and $\xi_{i}: G \rightarrow S$ be a selection of $\lambda$ for $i \in \mathbb{N}$. Write $g_{i}(t, s)=\xi_{i}(\lambda(t) \star \lambda(s))$ for $i \in \mathbb{N}, t, s \in S$. Then it is easy to check that (15) holds for every $f \in X^{S}$ (with $N=1$ and $\varepsilon_{l}(t, s)=0$ for $\left.t, s \in S\right)$.

(v) We finish with a somewhat trivial example. Namely, let $\lambda_{i}: S \rightarrow S$ and $g_{i}(t, s)=\lambda_{i}(t)$ for $i \in \mathbb{N}, t, s \in S$. Then (15) holds with $N=1$ and $\varepsilon_{l}(t, s)=\left\|\sum_{i, j=1}^{l} a_{i} a_{j}\left[f\left(\lambda_{i}(t)\right)-f\left(\lambda_{i}\left(\lambda_{j}(t)\right)\right)\right]\right\|$ for $l \in \mathbb{N}, t, s \in S$.

Finally, from Theorems 1 and 2 we immediately obtain the following corollary.

Corollary 3. Let $X$ be locally compact, $T=S, N \in \mathbb{N}$, conditions (3) and (15) be valid with suitable $\varepsilon_{l}$, and $\delta>0$. Suppose that $f \in \mathcal{F}$ and $h: S \rightarrow \mathbb{K}$ satisfy

$$
\left\|\sum_{i=1}^{\infty} a_{i} f\left(g_{i}(t, s)\right)-h(t) f(s)\right\| \leq \delta, \quad \forall t, s \in S .
$$

Then one of the following three conditions holds.

(i) $f$ and $h$ satisfy the functional equations

$$
\begin{gathered}
\sum_{i=1}^{\infty} a_{i} h\left(g_{i}(t, s)\right)=h(t) h(s), \\
\sum_{i=1}^{\infty} a_{i} f\left(g_{i}(t, s)\right)=h(t) f(s)
\end{gathered}
$$

and there is $M \in \mathbb{N}$ with $a_{j} h\left(g_{j}(t, s)\right)=0$ and $a_{j} f\left(g_{j}(t, s)\right)=0$ for $t, s \in S, j>M$.

(ii) $f$ is unbounded, $h$ is a bounded solution of Eq. (28) with $|h(t)| \leq \sum_{i=1}^{\infty}\left|a_{i}\right|$ for $t \in S$, and there is $M \in \mathbb{N}$ with $a_{j} h\left(g_{j}(t, s)\right)=0$ for $t, s \in S, j>M$.

(iii) $f$ and $h$ are bounded and, in the case $a:=\sum_{i=1}^{\infty}\left|a_{i}\right|<\infty,|h(t)| \leq a+\frac{\delta}{C}$ for $t \in S$, where $0<C:=\sup _{t \in S}\|f(t)\|$.

Remark 6. It seems that, in the general situation, we cannot expect much more information on $f$ in statement (ii) of Corollary 3, statement (i) of Theorem 2 , and in Theorem 1 for bounded $h$. For instance, if $s_{0} \in S, h(t)=0$ and $g_{1}(t, s)=s_{0}$ for $s, t \in S$, then (3) and (15) are valid with $a_{j}=0$ (and any $g_{j}$ ) for $j>1$ and every function $f: S \rightarrow X$ satisfies (27) with $\delta=\left\|a_{1} f\left(s_{0}\right)\right\|$.

However, in some special cases we can get a further characterization of $f$; for some details see, e.g., $[12,21,22,36]$. We will discuss that problem in subsequent publications. 
Remark 7. The functions $g_{j}$, given in Example 2(i), satisfy condition (3), as well; the same applies to Example 2(iii), provided the semigroup $S$ is commutative. Furthermore, one can easily check that also the first pair of functions $g_{1}, g_{2}$, defined in Example 2(ii), fulfils (3) (with $a_{1}=a_{2}$ and $a_{j}=0$ for $j>2$ ).

Some information on functional equations similar to (3) can be found, e.g., in $[23,24]$.

\section{Acknowledgments}

Bing Xu has been supported by NSFC \#11101295.

Open Access. This article is distributed under the terms of the Creative Commons Attribution License which permits any use, distribution, and reproduction in any medium, provided the original author(s) and the source are credited.

\section{References}

[1] Aczél, J.: Lectures on Functional Equations and Their Applications. Academic Press, London (1966)

[2] Badora, R.: Note on the superstability of the Cauchy functional equation. Publ. Math. Debr. 57, 421-424 (2000)

[3] Badora, R., Chmieliński, J.: Decomposition of mappings approximately inner product preserving. Nonlinear Anal. 62, 1015-1023 (2005)

[4] Badora, R., Ger, R. : On some trigonometric functional inequalities. In: Daróczy, Z., Pales, Z. (eds.) Functional Equations-Results and Advances, pp. 3-15. Kluwer Academic Publishers, New York (2002)

[5] Baker, J.: The stability of the cosine equation. Proc. Am. Math. Soc. 80, 411-416 (1980)

[6] Baker, J., Lawrence, J., Zorzitto, F.: The stability of the equation $f(x+y)=$ $f(x) f(y)$. Proc. Am. Math. Soc. 74, 242-246 (1979)

[7] Batko, B.: Stability of Dhombres' equation. Bull. Aust. Math. Soc. 70, 499-506 (2004)

[8] Brzdȩk, J.: On solutions of a generalization of the Reynolds functional equation. Demonstr. Math. 41, 859-868 (2008)

[9] Cholewa, P.W.: The stability of the sine equation. Proc. Am. Math. Soc. 88, 631634 (1983)

[10] Chudziak, J.: Stability of the generalized Goląb-Schinzel equation. Acta Math. Hung. 113, 133-144 (2006)

[11] Chudziak, J., Tabor, J.: On the stability of the Goląb-Schinzel functional equation. J. Math. Anal. Appl. 302, 196-200 (2005)

[12] Czerwik, S.: Functional Equations and Inequalities in Several Variables. World Scientific, London (2002)

[13] Dubreil-Jacotin, M.L.: Propriétés algébriques des transformations de Reynolds. C. R. Acad. Sci. Paris 236, 1950-1951 (1953)

[14] Förg-Rob, W., Schwaiger, J.: On the stability of some functional equations for generalized hyperbolic functions and for the generalized cosine function. Result. Math. 26, 274280 (1994)

[15] Forti, G.L.: Hyers-Ulam stability of functional equations in several variables. Aequ. Math. 50, 143-190 (1995)

[16] Ger, R., Šemrl, P.: The stability of the exponential equation. Proc. Am. Math. Soc. 124, 779-787 (1996) 
[17] Hyers, D.H.: On the stability of the linear functional equation. Proc. Natl. Acad. Sci. USA 27, 222-224 (1941)

[18] Hyers, D.H., Isac, G., Rassias, Th.M.: Stability of Functional Equations in Several Variables. Birkhäuser, London (1998)

[19] Jabłoński, W.: Stability of homogeneity almost everywhere. Acta Math. Hung. 117, 219$229(2007)$

[20] Jung, S.-M.: On the superstability of the functional equation $f\left(x^{y}\right)=y f(x)$. Abh. Math. Sem. Univ. Hambg. 67, 315-322 (1997)

[21] Jung, S.-M.: Hyers-Ulam-Rassias Stability of Functional Equations in Mathematical Analysis. Hadronic Press, Palm Harbor (2001)

[22] Kim, G.H.: The stability of d'Alembert and Jensen type functional equations. J. Math. Anal. Appl. 325, 237-248 (2007)

[23] Krapež, A.: Generalized associativity on rectangular quasigroups. In: Daróczy, Z., Palés, Zs. (eds.) Functional Equations-Results and Advances (Adv. Math. Dordr.), 3, pp. 335-349. Kluver Academic Publishers, Dordrecht (2002)

[24] Maksa, Gy.: The generalized associativity equation revisited. Rocz. Nauk. Dydakt. Prace Mat. No. 17, 175-180 (2000)

[25] Matras, Y.: Sur l'équation fonctionnelle: $f(x \cdot f(y))=f(x) \cdot f(y)$. Acad. R. Belg. Bull. Cl. Sci. (5) 55, 731-751 (1969)

[26] Moszner, Z.: General theory of the translation equation. Aequ. Math. 50, 17-37 (1995)

[27] Najdecki, A.: On stability of a functional equation connected with the Reynolds operator. J. Inequal. Appl. 2007(79816) (2007)

[28] Székelyhidi, L.: The stability of D'Alembert type functional equation. Acta Sci. Math. (Szeged) 44, 313-320 (1982)

[29] Székelyhidi, L.: On a theorem of Baker, Lawrence nad Zorzitto. Proc. Am. Math. Soc. 84, 95-96 (1982)

[30] Székelyhidi, L.: An abstract superstability theorem. Abh. Math. Sem. Univ. Hambg. 59, 81-83 (1989)

[31] Székelyhidi, L.: The stability of the sine and cosine functional equations. Proc. Am. Math. Soc. 110, 109-115 (1990)

[32] Tabor, J.: Stability of the Cauchy type equation in $\mathcal{L}_{p}$ norms. Result. Math. 32, 145$158(1997)$

[33] Tabor, J.: Stability of the Cauchy equation with variable bound. Publ. Math. Debr. 51, 165-173 (1997)

[34] Tabor, J., Tabor, J.: Homogeneity is superstable. Publ. Math. Debr. 45, 123-130 (1994)

[35] Trif, T.: On the superstability of certain functional equations. Demonstr. Math. 35, 813$820(2002)$

[36] Tyrala, I.: Stabilność trygonometrycznych równań funkcyjnych (Polish: Stability of trigonometric functional equations). Ph.D. Thesis, Silesian University, Katowice (2006)

[37] Wilson, W.H.: On certain related functional equations. Bull. Am. Math. Soc. 26, 300$312(1919)$

Janusz Brzdęk

Department of Mathematics

Pedagogical University

Podchorążych 2, 30-084 Kraków

Poland

e-mail: jbrzdek@up.krakow.pl 
Adam Najdecki

Faculty of Mathematics and Natural Sciences

University of Rzeszów

Rejtana 16A, 35-959 Rzeszow

Poland

e-mail: najdecki@ur.edu.pl

Bing Xu

Department of Mathematics

Sichuan University

Chengdu, 610064 Sichuan

People's Republic of China

e-mail: bxu@scu.edu.cn

Received: November 16, 2013 